\section{Anaesthetic Contamination}

SIR,-Your leading article (24 March, p. 693) draws attention to the statistical evidence suggesting an association between various toxic effects and pollution of the atmosphere with volatile anaesthetics in operating theatres and recovery areas. However, for those not fully familiar with this subject a number of points require clarification.

The implication that the problem is primarily related to halothane may be misleading Nitrous oxide should receive equal attention. Anaesthesia with halothane is almost always accompanied by nitrous oxide and in addition most "non-halothane anaesthetics" will have nitrous oxide as a constituent. Nitrous oxide is thus probably the most commonly administered anaesthetic in use today. It is present in the atmosphere of operating areas and has been detected in the expired air of personnel working in these areas. In animals nitrous oxide is teratogenic, as are all the volatile anaesthetics, at anaesthetic concentrations, and circumstances have been suggested under which nitrous oxide could be carcinogenic $c^{1}$. Furthermore, the original report $^{2}$ drawing attention to teratogenic effects in theatre personnel was made in operating theatres where nitrous oxide and ether were the atmospheric contaminations; halo thane was not in use. As nitrous oxide does not appear to be adsorbed by activated charcoal, Dr. R. S. Vaughan and his colleagues (24 March, p. 727) may be able to carry out an interesting clinical trial by using their Cardiff Aldasorber to remove halothane from the atmosphere.

With regard to halothane, although the original work by Cascorbi et al. ${ }^{4}$ did suggest that halothane stimulated its own metabolism, their more recent study 5 shows a wide scatter in both anaesthetists and nonanaesthetists; the evidence that halothane influences its own metabolism is not convincing. Even so, at present all available evidence shows that neither normal nor "abnormal" metabolism results in the formation of hepatotoxic metabolites, either in man or in many animal species. ${ }^{6}$ Furthermore, the 20-year survey of causes of death in American anaesthetists ${ }^{7}$ to which you refer did not demonstrate an increased incidence of liver disease as compared with the general population. As to removing the risk of anaesthetic contamination, your statement that this "depends in the first place on the provision of large operating and recovery rooms with air conditioning that changes the air several times an hour" is not consistent with the fact that most of the surveys on pollution have been carried out in well-ventilated, airconditioned operating areas in North America.

Since reduction of atmospheric pollution in operating areas is not straightforward, it is essential to establish that a problem exists in relation to toxic effects on personnel, particularly as the most obvious available method of reducing pollution (avoidance of inhalational anaesthetics) may be detrimental to patients undergoing general anaesthesia.-I am, etc.,

LEO STRUNIN

King's College Hospital,

1 Johnson, E. B., Lancet, 1972, 2, 824. Vaisman, A. I., Eksperimental 'naia Khirurgiya
Anesteziologiya, 1967, 12, no. 3, 44.

Vaughan, R. S., Mapleson, W. W., and Mushin
4 Cascorbi, H. F., Blake, D. A., and Helrich, M., 5 Cascorbi, H. F, Blake, D. A., And Helrich, M., in Cellular Biology and Toxicity of Anesthetics, ed. B. R. Fink

6 Strunin, L., and Simpson, B. R., British fournal of Anaesthesia, $1972,44,919$.

Eckenhoff, J. E., Anesthesiology, 1968 29,", 565 .

\section{Serum Alkaline Phosphatase and Rickets}

SIR,-The recent report by Dr. W. T. Cooke and others (10 February, p. 324) requires comment since its provocative conclusions are not supported by the results presented.

Firstly, the authors chose to study an age range for each sex (14-17 years) during which the rapid changes in serum alkaline phosphatase level associated with puberty are at their peak in boys while almost complete in girls, ${ }^{1}$ who mature two years earlier. ${ }^{2}$ It would therefore have been more logical to screen girls of 12-15 and boys of 14-17 if their aim was to detect "biochemical rickets" during puberty. Even then, yearly age corrections within each group would be important.

Secondly, their supposed normal range of alkaline phosphatase levels for the three-year period, 8-17 K.A. units in girls and 9-23 K.A. units in boys, appears to be stated without supporting evidence and in apparent contradiction to the data they present. Despite their analytucal method for alkaline phosphatase, which gives results at least $19 \%$ higher than those of other workers, they still adopt an arbitrary upper limit of 30 K.A. units and state that the majority of children with values above this limit probably have rickets. The simple calculation which follows from this is that the majority of a proportion approaching $41 \%$ of all schoolchildren in the age group studied (14-17 years) have the disease. This conclusion, certainly with regard to schoolchildren of Caucasian ancestry, is perhaps more likely to be based on the false premises with which they started. In order to have some value, any screening test must at least reveal some proportion of clinical disease among its positive readings. Fig. 2 of the report (the legend to which is, incidentally, faulty) suggests that about $40 \%$ of Caucasian boys had serum alkaline phosphatase levels above 30 K.A. units; the fact that none of these children had radiological evidence of rickets surely raises questions about the reliability of the authors' definition and about the whole interpretation of their results.

It may not be valid for them to assume that values in healthy children have a normal gaussian distribution, since the data of one of us (J.M.R.) indicate a log-normal distribution of alkaline phosphatase levels above the age of 11 . Allowing for the $19 \%$ higher phosphatase values obtained by their laboratory method, their actual mean levels over the limited age range studied (very much higher than the supposed normal range which they quote) agree reasonably closely with the results obtained by one of us in a study of London schoolchildren of Caucasian ancestry. 1

They stress the importance of low urinary calcium and high hydroxyproline excretion in their diagnostic procedure, while their own results (figs. 3 and 4) emphasize the complete lack of correlation between these measurements and radiological evidence of rickets.

Finally, the report lays great store by the effect of a large daily dose $(1.25 \mathrm{mg})$ of calciferol in lowering the highest phosphatase values found among healthy children. The effects of vast doses of calciferol during the normal puberty growth spurt have not been previously studied; however, it may be just as valid to draw the exactly opposite conclusion from the data presented. The authors' fig. 5 shows that healthy boys maintained alkaline phosphatase levels in the region of 40 K.A. units during the whole period from 1 to 6 months following an entirely unphysiological dose of calciferol (125 times the usually recommended antirachitic dose of $400 \mathrm{IU} /$ day). On the authors' criteria, most of these children would therefore still have rickets. Thus the "rickets" from which these children are supposed to be suffering could not even be cured by its specific therapy given in overdosage.

The problem of rickets and osteomalacia among our immigrant Asian community has been recently stressed and need not be underestimated. A proper understanding and solution of these problems may not be helped by confusing them with physiological changes at puberty which the present report does nothing to distinguish.-We are, etc.,

T. C. B. StaMP J. M. ROUND

University College Hospital Medical School, London W.C.I

1 Round, J. M., British Medical fournal, 1972, 3,114 Tanner, J. M., Growth at Adolescence, 2nd edn.
Oxford, Blackwell, 1962 .

\section{Hyperactive Children}

SIR,-It is surprising indeed that today, when in adults the various forms of depression and defences against depression are continually before the general practitioner, there is no mention in your leading article (10 February, p. 305) to the relationship of depression and mania in children to hyperactivity. It was many years ago that the late $D$. W. Winnicott, the paediatric psychoanalyst who added so much to our understanding of ways of making contact with children, disoussed the frequency of manic or hypomanic activity as a defence against depression in children.

The constant, restless, seemingly purposeless activity, the impulsiveness, the reduced attention span, the distractability, the inconsistency, the emotional immaturity and irritability, and the poor judgement which you describe as part of the picture of the hyperactive child are part of the picture of mania at any age. Unrecognized sleeplessness is often present as well. These symptoms may be due to many causes; surely by now we should all be alert to the possibility that one of these causes is a defence against depression. The presence of more or less conscious wondering whether one should give in to depression or "fight it off" by overactivity and cheerfulness is not confined to adults.

When manic excitement is considered as a possibility, then in interviewing the child one is much more apt to take a history which will include the possibility of the child being chronically depressed. In the simplest examples the child may be surprisingly ready 